\title{
Video Saurus System: Movement Evaluation by a Genetic Algorithm
}

\author{
Giuseppe Mastronardi, Vitoantonio Bevilacqua \\ D.E.E., POLITECNICO di Bari, Via E. Orabona, 4 \\ 70125 BARI - Italy \\ Email: mastrona@poliba.it, bevilacqua@poliba.it
}

\begin{abstract}
In this paper we have addressed the problem of adopting in a combined way a genetic algorithm [3] and the Hough Transform [1,2] for implementing an auto tracking method in a video conference system. By applying this method we have been able to track an object that moves slowly following quite parallel trajectories. The proposed algorithm considers just the shape of the object to be tracked and a priori known as a template, without taking into account other several characteristics of images like colour or texture. Because the implementation of Hough transform is a problem of maximising a function with particular constraints, and each run of evaluating Hough transform is time consuming, in this paper we have adopted a particular genetic algorithm to evaluate the rectangular region in which evaluate the Hough Transform 16]. The genetic algorithm used elitistic strategy, fitness sharing [5], mating restriction [4], adaptive rate of mutation and adaptive rate of cross-over with a double crosspoint/3\%. Moreover, in this paper, since the evaluation of Hough Transform is very time consuming, we have addressed the strategy of dividing the whole scene in different shorter window in order to partition the evaluating load on parallel DSP.
\end{abstract}

\section{THE GENERALISED HOUGH TRANSFORM}

In this paper we have considered just the shape of the object to be tracked without taking into account other several characteristics of images like colour or texture. The gray level image captured by the viedocamera has been filtered using the Sobel's filter in order to detect the edges and then the obtained image has been binaryzed using the mean value of the edge images as threshold. The Hough Transform is a technique which, using some curve representation, transforms a set of points defined over the image space to a set of points defined over some parameter space (known as Hough space). Hough Transform allows to detect several shapes as curves projecting them in a different domain built on the basis of their parametric representation. Owing of the problem of obtaining a parametric representation of curves we were interested in, it was not a trivial problem we have used a specialised technique proposed by Merlin and Farber, the well known Generalised Hough Transform (GHT) [1,2].

The GHT is a technique used to locate arbitrary curves in images, based on a 2 -dimensional template. Given a template, the first stage of the computation is to build an internal representation of the curves it contains. This representation is based on the position and orientation of each edge point with respect to some reference point. Using this representation, the GHT then looks for similar configurations of edge points in other input images. If such a configuration is found to exist, the template object is said to be localised, upon which the subsequent segmentation of the object from the background is trivial. In the following, the previous algorithm has been presented. Let us suppose that we are interested in detecting the curve $S$ shown in the left size of the Fig.1, which is our template, in the the right size of the same Fig.1, which is our captured image; both images are filtered using the same Sobel's filter.
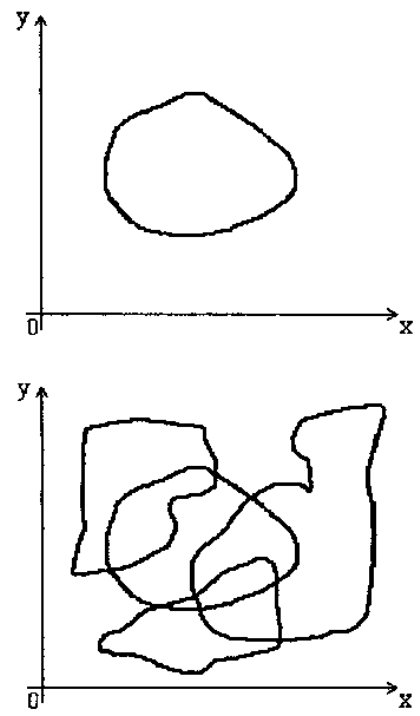

Fig. 1. Template and image for a Generalised Hough Transform application.

Points in Hough space represent particular instances of the curve in the image. Therefore, the strategy used by the GHT is to map sets of points from a particular instance of the considered curve, i.e. the template curve, to the point representing the curve in Hough space and, in effect, cause a peak to occur at that point. Once each point in the image plane has been considered, the Hough space is searched for the peak of maximum height. The co-ordinates of this peak in Hough space give the parameters which define the curve in the image plane.

The GHT begins with selecting a localisation point $A$ within a template of the idealised shape. Chosen a number of point $\mathrm{Pi}$ on the edge template we evaluate for each of them the distance to $A$, in terms of $\Delta x_{i}=x_{i}-x_{a}$, and $\Delta y_{i}=y_{i}-y_{a}$ and then for each point we stored the couple $\left(\Delta x_{i}, \Delta y_{i}\right)$ in the 
look-up table shown in the fig. 2. Let $H$ the so called accumulator array, the number of its rows equals the number of selected points on the edge template.

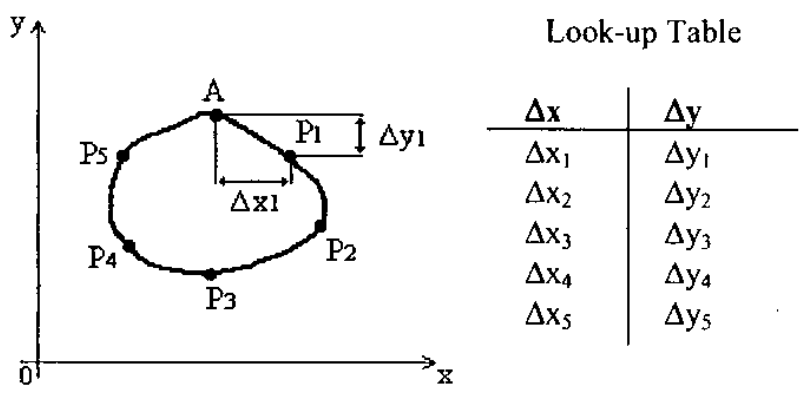

Fig. 2. S curve description using the look-up table.

Then, for each point $Q\left(x_{b}, y_{b}\right)$ belonging to the image we evaluate how many points having co-ordinates $\left(x_{b}+\Delta x_{i}\right.$, $\left.y_{b}+\Delta y_{i}\right) j=1 . . n$ are edge points and for each of them we increase $\mathrm{H}\left(\mathrm{x}_{\mathrm{b}}, \mathrm{y}_{\mathrm{b}}\right)$. With this strategy the problem of detecting the template $\mathrm{S}$ in the image has been regarded of a problem of maximising the $\mathrm{H}$ array.

\section{THE GENETIC ALGORITHM PROPOSED}

Genetic algorithm [3] are technique inspired by nature that in the past years have been adopted with success in many fields, where the problem to be investigated has been regarded as a problem of optimisation. In particular, we have been implementing several genetic strategy to different problems related to the image processing $[5,6,7]$. In the proposed GA each chromosome codes a rectangle with dimensions equal to the dimensions of the template to be searched, that explores randomly the video-camera image captured. As shown in fig. 3 each chromosome contains three different genes $\left(x_{0}, y_{0}, \alpha\right)$ and each gene is 16 bit binary coded. In particular, the binary code of the chromosome represents the rectangular window where the Hough Transform has to be evaluated, in fact, the three genes of the chromosome have coded the two vertices coordinates of the rectangle and its orientation.

The proposed fitness function has been designed to find out if a rectangle represented by the chromosome $x$ contains the template to be searched or does not, for this reason the fitness proposed is $f(x)=\max \left(H_{x}\right)$ where the accumulator array $H_{x}$ does not refer to the whole image but refers to the portion of the image contained in the rectangle individuated by the chromosome $x$.

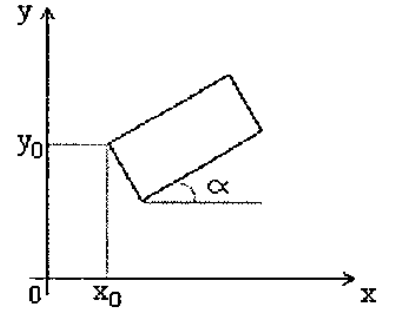

Fig. 3. geometric genes representation of the adopted chromosome

In order to speed up the convergence of the genetic algorithm in this paper we have presented the results obtained with a strategy called mating restriction. The goal of this strategy that presents analogy to several behaviour biologically plausible is to select parents with values of fitness quite equal to each other, estimating this similarity in terms of percentual compared to a fixed threshold. This algorithm selects different couples of parents for a number $n$ of times and then choose the parents which in that trials present the less percentual dissimilarity. At the same time for each generation a number of better individuals are copied in the next population, in order to avoid the lack of good performance. Moreover, in this algorithm a double crosspoint strategy has shown better results that a single point cross-over, and due to the multimodal characteristic of the fitness proposed we have also implemented the strategy of fitness sharing.

The fitness sharing that consists in re-scaling the fitness of each individual with a mechanism proportional to the number of similar individuals in the same generation, has shown good results avoiding the premature convergence to suboptimal solutions. In the previous sense the similarity of two individuals is a measure of the distance from $\mathrm{P} 1\left(\mathrm{x}_{10}, \mathrm{y}_{10}\right)$ and P2 $\left(x_{20}, y_{20}\right)$ in the image that is the search space, and it has been evaluated comparing this value to the dimension of the rectangle diagonal. Finally, better results in terms of convergence have been obtained using cross-over and mutation probability variable according to the following linear rules having fixed the range of that probability and the number Gen_Max of maximum generations:

$$
p_{\text {crossover }}=p_{\text {crossoverMAX }}-\frac{p_{\text {crossoverMAX }}-p_{\text {crossoverMIN }}}{G e n_{-} \operatorname{Max}} \times
$$

$\times$ (current_iteration_number)

$p_{\text {mutation }}=p_{\text {mutationMIN }}+\frac{p_{\text {mutationMAX }}-p_{\text {musationMIN }}}{G e n_{-} \text {Max }} \times$

$\times$ (current_iteration_number) 


\section{EXPERIMENTAL RESULTS}

In the fig. 4 are shown the experimental results obtained running the adopted genetic algorithm using a $433 \mathrm{MHz}$ Intel Pentium processor with the parameters presented in the following:

- Number of generations: 40

- Number of individuals: 30

- Number of individuals cloned with elitistic strategy: 10

- Range of cross-over probability : $0.60-1.00$

- Range of mutation probability: $0.01-0.30$

- Range of rotation : \pm 20 degrees

- Threshold of similarity for mating: $20 \%$

- Number of trials to find the best individual: 6

- Fitness sharing with threshold: 0.3

In the table 1 are presented the evaluation time, for processing the image shown in the left size of fig. 4, using an under sampling with factor 4 both in orizontal, both in vertical direction.

Table 1. Execution Time results

\begin{tabular}{|c|c|c|c|c|c|}
\hline $\mathbf{X}_{\mathbf{0}}$ & $\mathbf{Y}_{\mathbf{0}}$ & $\begin{array}{c}\boldsymbol{\alpha} \\
\text { (degree) }\end{array}$ & Generation & $\begin{array}{c}\text { Total time } \\
\text { consumed } \\
\text { (seconds) }\end{array}$ & $\begin{array}{c}\text { Time consumed to } \\
\text { fitness evaluation } \\
\text { (seconds) }\end{array}$ \\
\hline 15 & 3 & 3 & 0 & 21 & 21 \\
\hline 15 & 3 & 0 & 4 & 95 & 95 \\
\hline 22 & 1 & 0 & 12 & 266 & 261 \\
\hline 22 & 4 & -1 & 15 & 320 & 309 \\
\hline
\end{tabular}

\section{CONCLUSIONS}

The experimental results show that the evaluation time are directly linked to the dimension of the images and in particular to the number of edge points, the technique of fitness scaling is fundamental as the fitness function is multimodal, the elitistic strategy and the technique of mating restriction is particularly efficient for images that contain more than one object similar to the template, or for images corrupted by noise. The robustness of this method consists in tracking the right object both if in the scene captured there are a number of slow moving object different from the template, and both if in the scene there is the presence of noise. This device, named VideoSaurus System [3], is now experimentally employed in a tele-tutoring system and in a video monitoring system, used for the safety control of protected rooms. In particular, the strategy of fitness sharing has been very useful to speed-up the convergence of the proposed algorithm in such images where there are objects very similar to the template and in presence of noise.
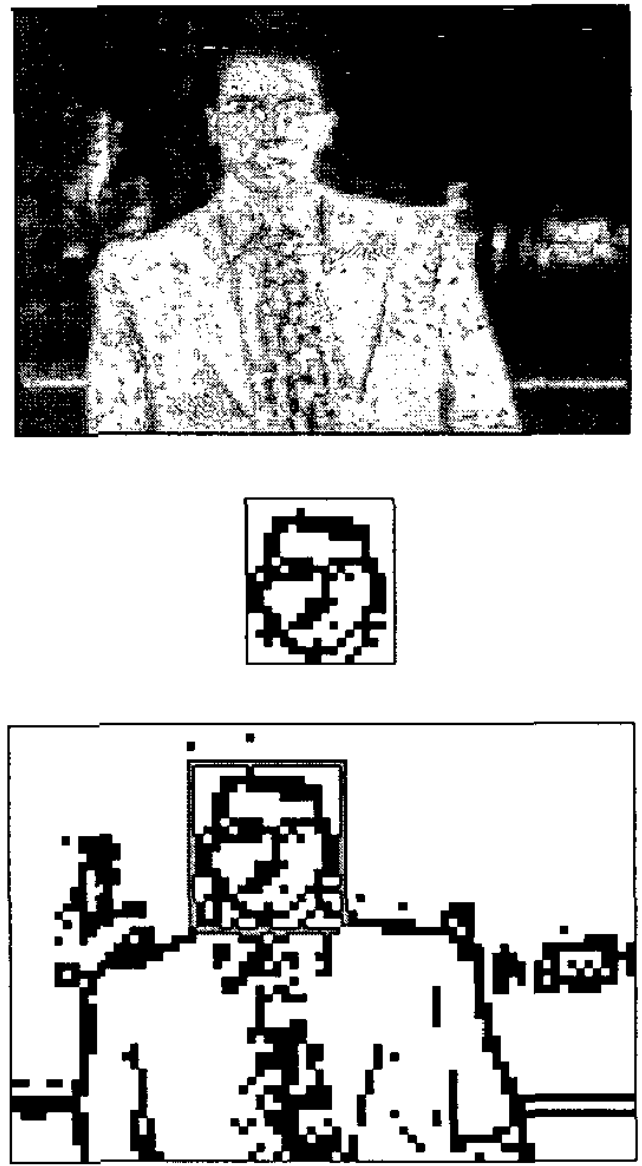

Fig. 4. the 256 gray level images captured by the video-camera, the template $(18 \times 20)$ and the edge images $(74 \times 52)$ showing the detected template.

\section{REFERENCES}

[1] N. Guil, E. L. Zapata, A New Invariant Scheme for the Generalized Hough Transform, August 1996.

[2] Philip M. Merlin, David J. Faber, "A Parallel Mechanism for Detecting Curves in Pictures". IEEE Trans. on Computer, pp. 96-98, 1975.

[3] Zbigniew Michalevicz, Genetic Algorithm + Data Structures = Evolution Programs, Springer, 1992.

[4] Carlos M. Fonseca and Peter J. Fleming, "Multiobjective Genetic Algorithms Made Easy: Selection, Sharing, and Mating Restriction". Proc. of the First International Conference on Genetic Algorithms in Engineering Systems: Innovations and Applications, pp. 42.52, Sheffield, UK, IEE, September 1995.

[5] P. Darwen and X. Yao, "A dilemma for fitness sharing with a scaling function". Proc. of 1995 IEEE Conference on Evolutionary Computation (ICEC'95), pp. 166-171, Perth, Australia, IEEE Press, 1995.

[6] G. Mastronardi, M. Arborea, R. Loscialpo, M. Semeraro, Video Saurus System, A project presented to Texas Instruments Challenge, 1999.

[7] V. Bevilacqua, A. Sappa, M. Devy, "Improving a genetic algorithm segmentation by means of a fast edge detection technique". Proc. of ICIP 2001, IEEE International Conference on Image Processing, pp. 754-757, Tessalonica, Grecia, October 2001. 